\title{
Toxicokinetic and Tissue distribution studies of Mercury in an Ayurvedic preparation- Shila Sindur
}

\author{
Challa Srinivas Reddy ${ }^{1 *}$, Umamahesh Balekari ${ }^{2}, V^{2}$ emu Priyanka ${ }^{3}$, Jinugu Vidya Sagar ${ }^{4}$
}

1. Department of Pharmacognosy, Vaagdevi college of Pharmacy, Hanamkonda, India

2. Department of Pharmacognosy, University College of Pharmaceutical Sciences, Kakatiya University, Hanamkonda, Andhrapradesh, India

3. Department of Pharmacology, Vaagdevi College of Pharmacy, Hanamkonda, Andhrapradesh, India.

4. Relisys Medical Devices Ltd, Hyderabad, Andhra Pradesh India.

\begin{abstract}
Plan: Shila sindur, an ayurvedic preparation containing mercury as chief ingredient was investigated for toxicokinetics and tissue distribution studies in laboratory animals.

Methodology: Shila Sindur at three doses i.e., $50 \mathrm{mg} / \mathrm{kg}, 300 \mathrm{mg} / \mathrm{kg}$ and $1000 \mathrm{mg} / \mathrm{kg}$ was studied. Single and repeated dose administration was used for toxicokinetics and tissue distribution studies, respectively and evaluated by using toxicokinetic and tissue distribution parameters.

Outcome: Toxicokinetics studies revealed low plasma clearance with high half life, this correlates with higher affinity of mercury to plasma proteins. Higher doses showed wide distribution by increased area under curve and volume of distribution. A dose dependent elevation in mercury concentrations and organ damage was found in kidneys followed by liver, lungs, spleen and brain. At higher doses, shila sindur found toxic, at moderate doses stringent monitoring was recommended for use. Present study concludes that low dose Shila sindur was found safe in terms of toxicological and tissue distribution patterns.
\end{abstract}

Key words: Shila sindur, toxicokinetics, tissue distribution, mercury

\section{INTRODUCTION}

Ayurvedic medicine is a traditional system native to India and has been positioned as mainstream system of medicine since thousands of years. Globally, this system of medicine gained significance as an alternative and complementary medicine ${ }^{1,2}$. This system uses natural plant based medicines and minerals including sulfur, arsenic, lead, copper and gold are often added to formulations with the belief that these metals are essential components of vital molecules within the human body ${ }^{3}$.

For Correspondence: challa_cnu@yahoo.com

Contact: +91-9885108060

Hygeia.J.D.Med. Vol.6 (1), April 2014@ 2014 all rights reserved.

Hygeia journal for drugs and medicines, 2229 3590, 09756221

D.O.I: 10.15254/H.J.D.Med.6.2014.125 
Indian Ayurvedic system and herbal medicines have attracted the global attention due to their slow and explicit cure (Anand Prem Rajan). Approximately $80 \%$ of India's 1 billion population use ayurveda ${ }^{4,5}$. This developing interest and usage raised up safety concerns about Ayurveda. Recent studies conducted by United States on this system of medicine found about $20 \%$ of ayurvedic treatments are containing toxic levels of heavy metals such as lead, mercury and arsenic ${ }^{6}$.

Rasashastra, an intergral part of Ayurveda, deals with drugs of mineral origin comprehensively. In traditional Ayurvedic medicine as part of Rasa Shastra, practioners add minerals to plants/animal origin drugs and claim that such medicines are safe, when they are properly purified and detoxified. According to 'The Ayurvedic Formulary of India' mercury and lead are the most widely used heavy metals. As these metals are potent nephrotoxic, hepatotoxic, neurotoxic and hematotoxic agents ${ }^{6,7}$. The toxicokinetics of these metals in ayurvedic preparations is geared up. Since there are no clear standards and recommendations are defined for metals contamination in ayurvedic preparations. In adequate scientific studies and clinical trials of many ayurvedic products lead to such circumstances ${ }^{6}$.

This study was aimed to investigate toxicokinetic parameters, to detect and estimate mercury accumulation in various organs on a 28 days intake of Shila sindur, a herbo-mineral preparation composed of mercury, sulphur and arsenic disulfide (realgar) along with aloe vera. Shila sindur is indicated for all types of skin disorders, skin diseases associated with itching, skin conditioning and other diseases of infectious origin like fever, abscess, gonorrhea, cardio protection and neuro protection ${ }^{8}$.

\section{MATERIALS AND METHODS}

\section{Reagents and chemicals}

Shila sindur was procured from the local market. Sodium Carboxy Methyl Cellulose, haemotoxylin, eosin, mercuric chloride, 2-mercaptoethanol and HPLC grade methanol were procured from SD fine chem., Mumbai. All reagents and chemicals used were analytical grade.

\section{Animals}

Male adult Wistar albino rats age of 6-7 weeks, weighing between 150 to 200g, were used in the study. They were procured from the Mahaveera enterprises (Reg no.146/1999 CPCSEA) Hyderabad, Andhra Pradesh. The animals were housed at CPCSEA Approved (Reg.no.1047/ac/07 CPCSEA) animal house of Vaagdevi college of Pharmacy. They were maintained under standard laboratory conditions at an ambient temperature of $25 \pm 2^{\circ} \mathrm{C}$ and $50 \pm 15 \%$ relative humidity, with a 12-h light/12-h dark cycle. Rats were fed with a commercial pellet diet (Hindustan lever Pvt. Ltd. Bombay, India.) and water ad libitum. They were fasted for $18 \mathrm{~h}$ prior to the experiment and during the experiment; the food and water were withdrawn. The animal experiments were performed after prior approval of the study protocol by the Institutional Animal Ethics Committee (1047/ac/07/CPCSEA, Dated 24/04/2007) of Vaagdevi college of Pharmacy, Warangal. Ethical norms were strictly followed during all experiments. 


\section{Toxicokinetic studies}

Toxicokinetic studies were conducted as per OECD guideline $417^{9}$. The animals were grouped into 3 groups of 6 animals each. The doses were set as $50 \mathrm{mg} / \mathrm{kg}$ (low), $300 \mathrm{mg} / \mathrm{kg}$ (medium) and $1000 \mathrm{mg} / \mathrm{kg}$ (High). Shila sindur was administered orally (suspension with $0.1 \%$ Sodium CMC) to overnight fasted rats (OECD 417). Blood samples were taken at $1 \mathrm{hr}, 2 \mathrm{hr}, 4 \mathrm{hr}, 6 \mathrm{hr}, 12 \mathrm{hr}, 24 \mathrm{hr}$ and $48 \mathrm{hr}$ by retro-orbital puncture.

\section{Tissue Distribution Studies}

Tissue distribution studies guidelines were adopted from OECD guideline $417^{9}$. Animals were divided into six groups based on the dose and frequency of administration of drug i.e., High/repeated doses, High/single dose, Medium/repeated doses, Medium/single dose, Low/repeated doses, Low/single dose). In each group 6 animals were taken. In repeated doses groups, the shila sindur was administered for 28 days. After $24 \mathrm{hrs}$ (for single dose) and $28^{\text {th }}$ day (for repeated doses), the animals were sacrificed. Liver, lungs, kidneys, brain and spleen were collected and then weighed for wet weight and homogenized in phosphate buffer saline (PBS) of $\mathrm{pH}$ 7.4. The tissue homogenates were centrifuged at approximately 4000 rpm for $20 \mathrm{~min}$ and stored at $-20^{\circ} \mathrm{C}$ until analysis.

Clinical Bio-Chemistry Parameters

Clinical biochemical parameters like hematological parameters assessed by using Erba Heamo Lab-8 Heamaolog analyser ( US Tech Inc, Washington MD, USA); liver function parameters i.e., SGOT and SGPT; Kidney function by assessing Serum Creatinine were conducted by using respective diagnostic kits (Crest Biosystems).

\section{Histopathology}

The rats were sacrificed by cervical dislocation. Complete necropsies were carried out on all animals. Tissues were preserved in $10 \%$ neutral buffered formalin. The tissues examined were brain, lungs, liver, spleen and kidney. Tissues were subjected to microscopic examination by embedding in paraffin wax, sectioned at five micrometers thickness by using Microtome (Rofin, Switzerland) and stained with haemotoxylin and eosin. Scanning Electron Microscopy (SEM) images were obtained for all the tissues (SEM- Joel, USA) to observe histopathology at various doses.

\section{Analysis}

The blood samples were analyzed using HPLC-ICP-MS. The kinetic parameters were estimated using Kinetica (version 5.0)

HPLC - ICP MS method validation for the presence of mercury

1. Equipment: Total mercury concentrations were determined using a Perkin Elmer SCIEX Elan DRC-e ICPMS (Table 1). Mercury species were determined using a Perkin Elmer series 200 HPLC pump with a Perkin-Elmer 3_mC8 $(33 \mathrm{~mm} \times 3 \mathrm{~mm})$ column coupled to a Perkin Elmer Sciex Elan 6000 ICPMS (Table 2). The lowest quantifiable mercury concentration was $0.004 \mu \mathrm{g} / \mathrm{ml}$. 


\section{Standards:}

A stock solution $(1000 \mathrm{mg} / \mathrm{l})$ of mercury (II) was prepared by dissolving the appropriate amount of mercuric chloride in de-ionized water. Stock solution was stored in airtight bottle and refrigerated. Working standards $[(10 \mathrm{ng} / \mathrm{ml}$ to $3 \mu \mathrm{g} / \mathrm{ml})$ and $(5,10,30,50,100 \mu \mathrm{g} / \mathrm{ml})\}$ were prepared daily from the stock solution by serial dilution using extracting solutions.

\section{Reagents:}

All reagents were analytical reagent grade and used without further purification. A 2-mercaptoethanol solution was prepared containing $0.5 \%(\mathrm{v} / \mathrm{v}) 2$-mercaptoethanol in $5 \%(\mathrm{v} / \mathrm{v})$ methanol and used as the extracting reagent and mobile phase for HPLC-ICPMS work.

\section{Measurement of Hg concentration in Rat tissues and sediments by 2-mercaptoethanol extraction:}

Freeze-dried samples $(0.2 \mathrm{~g})$ were weighed into $55 \mathrm{ml}$ polytetrafluroacetate (PTFE) digestion vessels (CEM, USA) with $5 \mathrm{ml}$ and $2.5 \mathrm{ml}$ of $0.5 \%(\mathrm{v} / \mathrm{v}) 2$-mercaptoethanol for rat tissues and sediment samples respectively. The vessels were heated in a microwave oven at $120^{\circ} \mathrm{C}$ for $15 \mathrm{~min}$. The extracts were transferred to acid washed $10 \mathrm{ml}$ polypropylene centrifuge tubes and centrifuged in an Eppendorf centrifuge 5804 for $20 \mathrm{~min}$ at $3000 \mathrm{rpm}$. For sediment samples, after the supernatant was separated, the extraction was repeated and extracts combined.

\section{HPLC-ICP-MS:}

All supernatants were filtered through Acrodisc LC 13-mm Syringe filters with 0.2_mPVDF membranes before analysis. Aliquots of extracts $(100 \mu \mathrm{l})$ were injected onto the HPLC-ICP-MS (Table 2). External calibration using the standards $0-100 \mu \mathrm{g} / \mathrm{l}$ was used. The chromatography package Turbochrom Navigator (Perkin Elmer, Australia) was used to quantify mercury species by peak area.

Table 1: Operating conditions for ICP-MS.

\begin{tabular}{lc}
\hline & Plasma conditions \\
\hline RF forward power & $1200 \mathrm{~W}$ \\
Plasma argon flow rate & $15 \mathrm{~L} / \mathrm{min}$ \\
Auxiliary argon flow rate & $1.2 \mathrm{~L} / \mathrm{min}$ \\
Nebulizer gas flow & $0.92 \mathrm{~L} / \mathrm{min}$ \\
\hline & Mass spectrometer settings \\
\hline Acquisition mode & Peak hopping \\
Isotopes monitored & $202 \mathrm{Hg}, 201 \mathrm{Hg}, 200 \mathrm{Hg}, 198 \mathrm{Hg}, 181 \mathrm{Ta}, 184 \mathrm{~W}, 103 \mathrm{Rh}, 115 \mathrm{In}, 159 \mathrm{~Tb}, 165 \mathrm{Ho}$ \\
Dwell time & $50 \mathrm{~ms}$ \\
Sweep time & $13.5 \mathrm{~s}$ \\
Sweeps per reading & 15 \\
Replicates & Total: 3 \\
Integration time & $750 \mathrm{~ms}$ per element \\
\hline
\end{tabular}


Table 2: Operating conditions for HPLC-ICP-MS.

\begin{tabular}{ll}
\hline Chromatography & \\
\hline HPLC column & PE C $8,3 \_\mathrm{m}(33 \mathrm{~mm} \times 3 \mathrm{~mm})$ \\
Mobile phase & $0.5 \%(\mathrm{v} / \mathrm{v}) 2$-mercaptoethanol in $5 \%(\mathrm{v} / \mathrm{v}) \mathrm{CH} 3 \mathrm{OH}, \mathrm{pH} 5.3$, \\
& flow rate, $1.5 \mathrm{ml} \mathrm{min}-1 ;$ temp, $25{ }^{\circ} \mathrm{C}$ \\
Sample volume & $100 \mu \mathrm{l}$ \\
Plasma conditions & \\
RF forward power & $1200 \mathrm{~W}$ \\
Plasma argon flow rate & $15 \mathrm{lmin}-1$ \\
Auxiliary argon flow rate & $1.21 \mathrm{~min}-1$ \\
Nebulizer gas flow & $0.841 \mathrm{~min}-1$ \\
Mass spectrometer settings & \\
Acquisition mode & Peak hopping \\
Isotopes monitored & $202 \mathrm{Hg}, 201 \mathrm{Hg}, 200 \mathrm{Hg}, 198 \mathrm{Hg}, 181 \mathrm{Ta}$ and $184 \mathrm{~W}$ \\
Dwell time & $100 \mathrm{~ms}$ \\
Sweeps per reading & 1 \\
Replicates & 1 \\
Readings & $500(6.52 \mathrm{~min})$ \\
\hline
\end{tabular}

\section{RESULTS AND DISCUSSION}

\section{A. Blood Hg profile at various doses:}

Table 3: Concentration of $\mathrm{Hg}$ in blood at different time points

\begin{tabular}{clcc}
\hline \multirow{2}{*}{ Time $(\mathrm{hrs})$} & \multicolumn{1}{c}{ Concentration $(\mu \mathrm{g} / \mathrm{ml})$ of $\mathrm{Hg}$ in Blood } \\
\cline { 2 - 4 } & \multicolumn{1}{c}{ 1000mg/kg } & \multicolumn{1}{c}{$300 \mathrm{mg} / \mathrm{kg}$} & 0 \\
\hline 0 & $4.128 \pm 0.341^{* *}$ & $3.451 \pm 0.124^{* *}$ & $2.480 \pm 0.241$ \\
2 & $12.015 \pm 0.651^{* * *}$ & $8.140 \pm 0.412^{* * *}$ & $4.178 \pm 0.176$ \\
4 & $5.018 \pm 0.125^{* *}$ & $4.312 \pm 0.125^{* *}$ & $2.451 \pm 0.270$ \\
6 & $3.871 \pm 0.345^{* *}$ & $2.871 \pm 0.315^{*}$ & $1.631 \pm 0.311$ \\
12 & $2.151 \pm 0.158^{* *}$ & $1.021 \pm 0.315$ & $0.810 \pm 0.134$ \\
24 & $1.891 \pm 0.135^{* *}$ & $0.984 \pm 0.105^{*}$ & $0.503 \pm 0.089$ \\
48 &
\end{tabular}

All values are expressed as Mean $\pm S D, n=4,{ }^{*}<0.05 ; * * p<0.01 * * * p<0.001$ when compared to $50 \mathrm{mg} / \mathrm{kg}$ dose group. Statistical Comparision was performed by using ANOVA coupled with Dunnet's multiple comparision test.

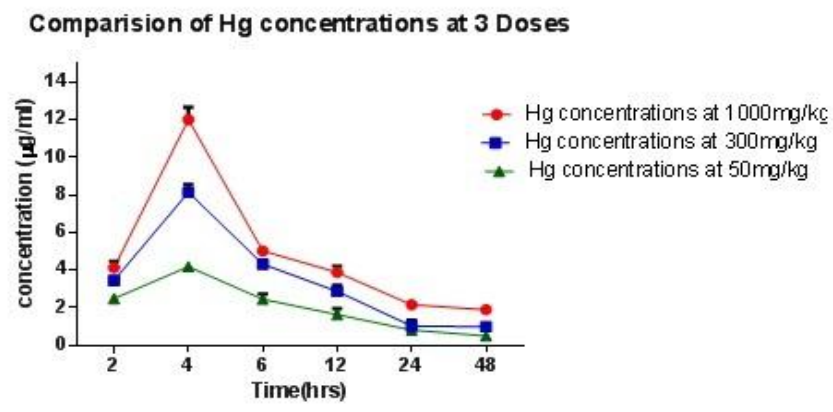

Fig 1: Graph for Concentration Vs Time Profiles 
C Srinivas Reddy et al.

B. Toxicokinetic parameters

Table 4: Toxicokinetic Parameters

\begin{tabular}{|c|c|c|c|c|c|}
\hline Sl. No & Parameter & Units & $1000 \mathrm{mg} / \mathrm{kg}$ & $300 \mathrm{mg} / \mathrm{kg}$ & $50 \mathrm{mg} / \mathrm{kg}$ \\
\hline 1 & $\mathrm{C}_{\max }$ & $\mu \mathrm{g} / \mathrm{ml}$ & $12.01 \pm 0.15^{* * *}$ & $8.14 \pm 2.19^{* *}$ & $4.17 \pm 0.30$ \\
\hline 2 & $\mathrm{~T}_{\max }$ & $\mathrm{hr}$ & $4.0 \pm 0.00$ & $4.0 \pm 0.00$ & $4.0 \pm 0.00$ \\
\hline 3 & HVD & $\mathrm{hr}$ & $3.11 \pm 0.19^{* *}$ & $4.588 \pm 0.80^{*}$ & $6.6 \pm 0.98$ \\
\hline 4 & AUC last & $\mu \mathrm{g} / \mathrm{ml} * \mathrm{hr}$ & $154.91 \pm 15.02^{* * *}$ & $103.093 \pm 9.25^{* * *}$ & $61.538 \pm 9.58$ \\
\hline 5 & AUC extra & $\mu \mathrm{g} / \mathrm{ml} * \mathrm{hr}$ & $83.52 \pm 6.37 * * *$ & $28.634 \pm 5.23 * * *$ & $13.784 \pm 3.61$ \\
\hline 6 & AUC tot & $\mu \mathrm{g} / \mathrm{ml} * \mathrm{hr}$ & $238.4 \pm 42.15^{* * *}$ & $131.727 \pm 16.29^{* * *}$ & $15.323 \pm 2.10$ \\
\hline 7 & $\mathrm{~T}_{1 / 2}$ & $\mathrm{hr}$ & $30.61 \pm 5.16^{* *}$ & $20.170 \pm 3.14^{*}$ & $18.994 \pm 4.13$ \\
\hline 8 & MRT & $\mathrm{hr}$ & $43.48 \pm 1.64 * *$ & $28.149 \pm 3.11 *$ & $25.983 \pm 4.15$ \\
\hline 9 & $\mathrm{~K}_{\mathrm{e}}$ & & $0.022 \pm 0.00015^{*}$ & $0.034 \pm 0.001$ & $0.036 \pm 0.0018$ \\
\hline 10 & $\mathrm{~V}_{\mathrm{d}}$ & $\mathrm{ml}$ & $158.45 \pm 44.8 * * *$ & $45.4 \pm 6.24^{* *}$ & $16 \pm 3.16$ \\
\hline 11 & $\mathrm{Cl}$ & $\mathrm{ml} / \mathrm{hr}$ & $3.48 \pm 0.62 * *$ & $1.54 \pm 0.25^{*}$ & $0.57 \pm 0.012$ \\
\hline
\end{tabular}

All values are expressed as Mean $\pm S D, n=5,{ }^{*} p<0.05 ; * * p<0.01 * * * p<0.001$ when compared to $50 \mathrm{mg} / \mathrm{kg}$ dose group. Statistical Comparision was performed by using ANOVA coupled with Dunnet's multiple comparision test. $\boldsymbol{C}_{\max }$ : Peak plasma Concentration; $\boldsymbol{T}_{\max }$ : Time to reach peak concentration, HVD: Half- Value Duration; AUC: Area under plasma concentration /time; $\boldsymbol{t}_{1 / 2:}$ terminal half life; MRT: Average mean residence time. $\boldsymbol{K}_{e}$ : Elimination rate constant; $\boldsymbol{V}_{d}$ : Volume of distribution, $\mathrm{Cl}$ : Clearance.

C. Tissue distribution studies

Table 5: Concentration $(\mu \mathrm{g})$ of $\mathrm{Hg}$ in different tissues

\begin{tabular}{|c|c|c|c|c|c|}
\hline Doses & Brain & Liver & spleen & lungs & Kidney \\
\hline High / repeated dose & $0.015 \pm 0.001$ & $0.514 \pm 0.003 * *$ & $0.514 \pm 0.002$ & $0.714 \pm 0.0021 * * *$ & $25.781 \pm 2.347 * * *$ \\
\hline High / single dose & $0.001 \pm 0.0003$ & $0.07 \pm 0.003$ & $0.003 \pm 0.0003$ & $0.09 \pm 0.0005 * *$ & $2.151 \pm 0.098^{*}$ \\
\hline Medium / repeated doses & $0.014 \pm 0.002$ & $0.505 \pm 0.145^{* *}$ & $0.505 \pm 0.07$ & $0.405 \pm 0.003 * * *$ & $6.215 \pm 1.23 * *$ \\
\hline Medium / single dose & $0.015 \pm 0.001$ & $0.05 \pm 0.002$ & $0.002 \pm 0.0001$ & $0.05 \pm 0.004 * *$ & $1.315 \pm 0.089$ \\
\hline Low / repeated doses & $0.012 \pm 0.004$ & $0.124 \pm 0.013^{*}$ & $0.0211 \pm 0.003$ & $0.012 \pm 0.002 *$ & $2.012 \pm 0.015^{*}$ \\
\hline Low / single dose & $<$ LLOQ & $0.015 \pm 0.0002$ & $<$ LLOQ & $0.001 \pm 0.0001$ & $0.129 \pm 0.005$ \\
\hline High / repeated dose & $0.015 \pm 0.001$ & $0.514 \pm 0.003 * *$ & $0.514 \pm 0.002$ & $0.714 \pm 0.0021 * * *$ & $25.781 \pm 2.347 * * *$ \\
\hline
\end{tabular}

All values are expressed as Mean $\pm S D, n=4, * p<0.05 ; * * p<0.01 * * * p<0.001$ when compared to Low single dose group. Statistical Comparision was performed by using ANOVA coupled with Dunnet's multiple comparision test.

C. Ratio of tissue - blood concentrations

Table 6: Ratio of tissue to blood concentrations at different doses

\begin{tabular}{|c|c|c|c|c|c|}
\hline Doses & Brain & Liver & spleen & lungs & Kidney \\
\hline High / repeated dose & 4.128 & 0.003634 & 0.124516 & 0.100291 & 0.172965 \\
\hline High / single dose & 3.84 & 0.00026 & 0.018229 & 0.000781 & 0.023438 \\
\hline Medium / repeated oses & 3.451 & 0.004057 & 0.146334 & 0.146334 & 0.117357 \\
\hline Medium / single dose & 2.93 & 0.005119 & 0.017065 & 0.000683 & 0.017065 \\
\hline Low / repeated doses & 2.48 & 0.004839 & 0.05 & 0.008508 & 0.004839 \\
\hline Low / single dose & 0.98 & 0 & 0 & 0 & 0.00102 \\
\hline High / repeated dose & 4.128 & 0.003634 & 0.124516 & 0.100291 & 0.172965 \\
\hline
\end{tabular}




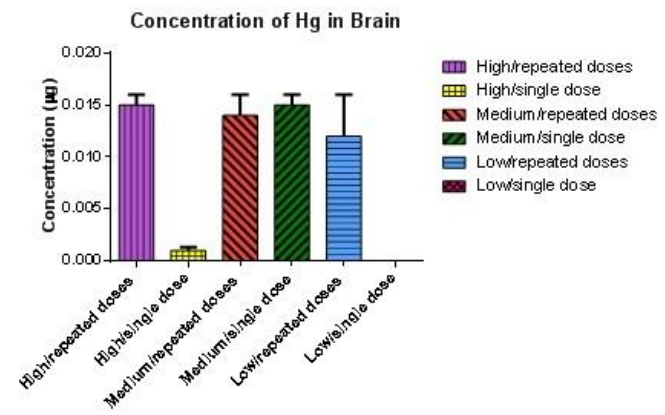

Fig 3: Concentration of Mercury in Brain

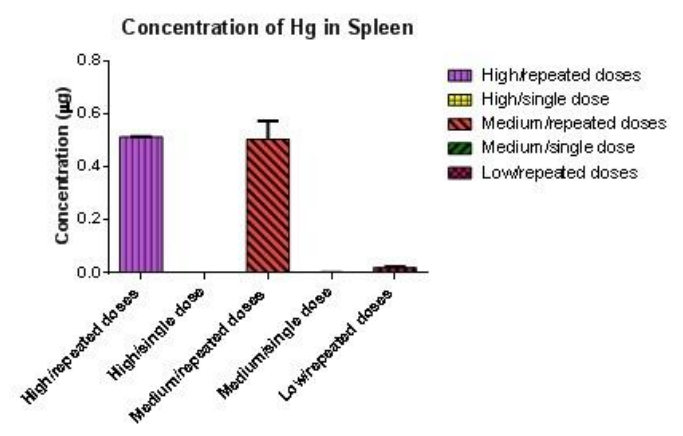

Fig 5: Concentration of Mercury in Spleen

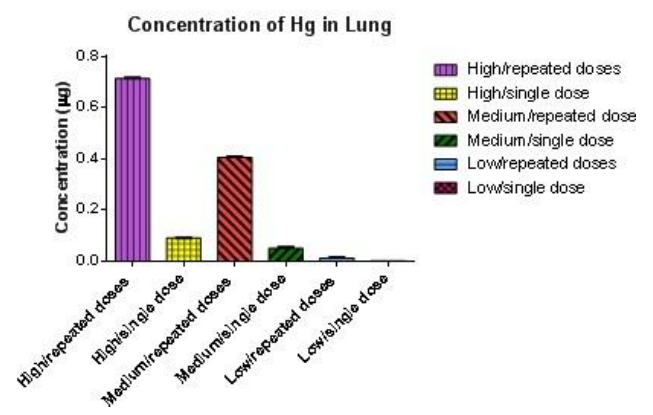

Fig 6: Concentration of Mercury in Lung

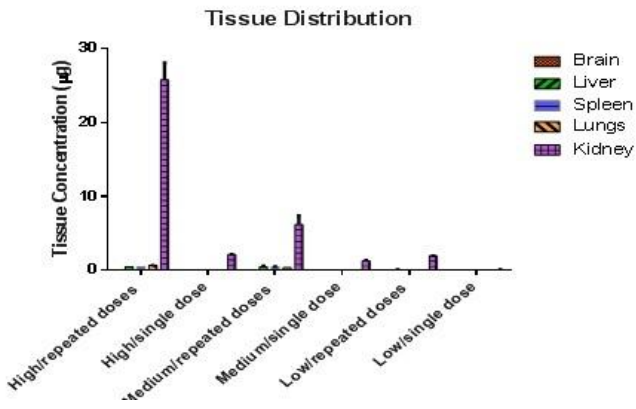

Fig 2: Tissue distribution

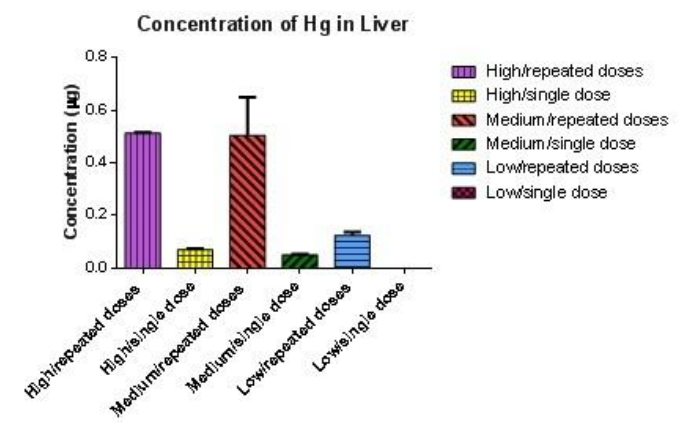

Fig 4: Concentration of Mercury in Liver

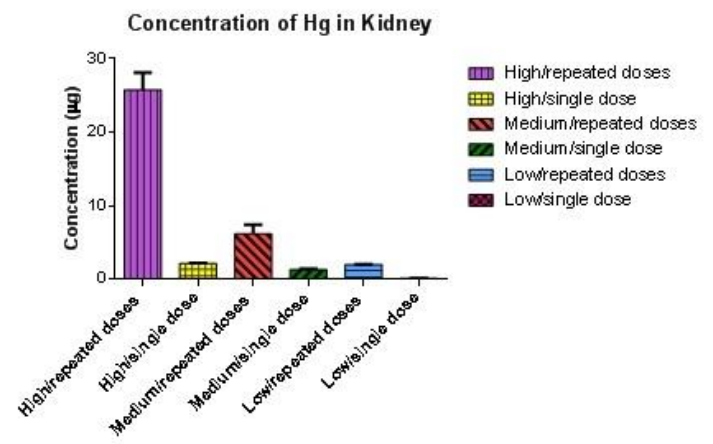

Fig 7: Concentration of Mercury in Kidney 
C Srinivas Reddy et al.

\section{Bio-chemical parameters}

\section{Complete blood picture}

Table 7: Complete blood picture of 28 day-treated animals at various doses

\begin{tabular}{cccc}
\hline Cells & 1000mg/kg & 300mg/kg & $50 \mathrm{mg} / \mathrm{kg}$ \\
\hline Haemoglobin(gms\%) & $13.9 \pm 0.18$ & $14.95 \pm 0.12 * * *$ & $14.95 \pm 0.07 * * *$ \\
R.B.C(m/cmm) & $4.98 \pm 0.03$ & $4.95 \pm 0.07$ & $4.96 \pm 0.04$ \\
Total count(TC) (m/cmm) & $4008.33 \pm 60.66$ & $3991.66 \pm 60.66$ & $7341.66 \pm 204.97 * * *$ \\
Neutrophils(\%) & $42.33 \pm 0.74$ & $35 \pm 1.63 * * *$ & $50 \pm 1.29 * * *$ \\
Lymphocytes(\%) & $53.5 \pm 1.25$ & $57 \pm 1.63 * * *$ & $43 \pm 1.29 * * *$ \\
Monocytes(\%) & $1.66 \pm 0.47$ & $4.5 \pm 0.5 * * *$ & $4.33 \pm 0.74 * * *$ \\
Eosinophils(\%) & $0 \pm 0$ & $0 \pm 0$ & $0 \pm 0$ \\
\hline
\end{tabular}

All values expressed as Mean $\pm \mathrm{SD},(\mathrm{n}=4) ;{ }^{*} \mathrm{p}<0.05$ significant, ${ }^{*} \mathrm{p}<0.01$ highly significant, $* * * \mathrm{p}<0.001$ very highly significant when compared with $50 \mathrm{mg} / \mathrm{kg}$ dose group. Statistical Comparision was performed by using ANOVA coupled with Dunnet's multiple comparision test.

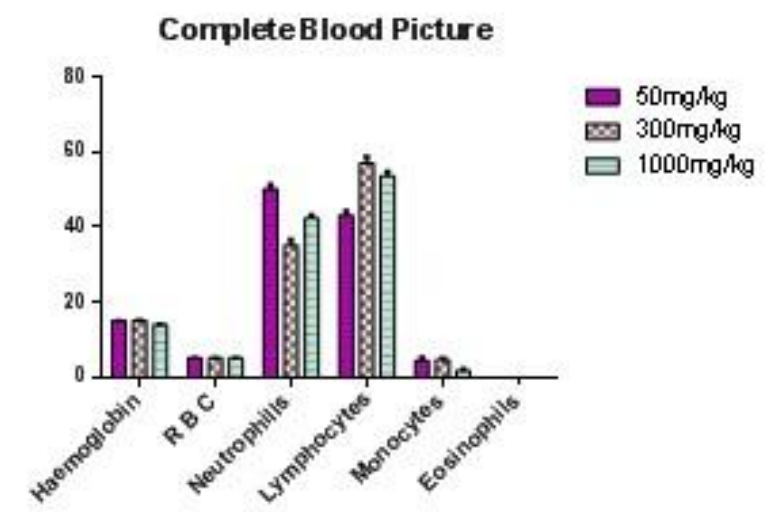

Total Count (CBP)

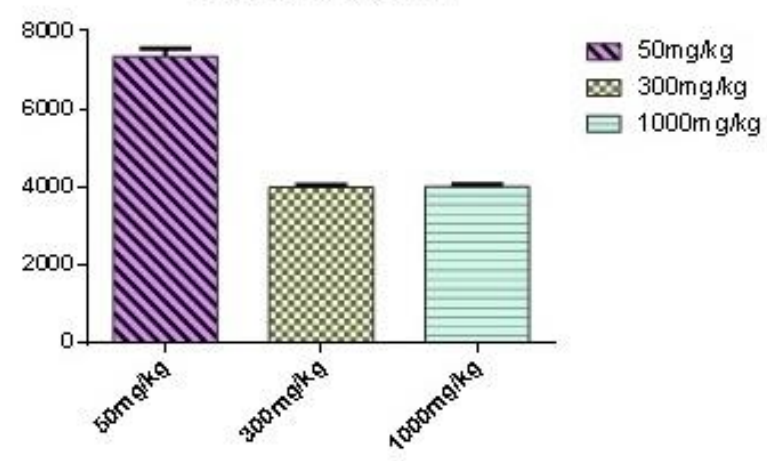


2. Liver function tests: SGOT and SGPT estimation

Table 8: Liver Function Tests of 28 day-treated animals at various doses

\begin{tabular}{ccc}
\hline Group & SGOT (U/L) & SGPT (U/L) \\
\hline $1000 \mathrm{mg} / \mathrm{kg}$ & $189.33 \pm 4.60^{* * *}$ & $178.50 \pm 5.02 * * *$ \\
$300 \mathrm{mg} / \mathrm{kg}$ & $93.33 \pm 2.42^{* *}$ & $80.66 \pm 1.69 * *$ \\
$50 \mathrm{mg} / \mathrm{kg}$ & $59.16 \pm 1.57 *$ & $68.83 \pm 2.26^{*}$ \\
Control & $38.23 \pm 1.20$ & $36.69 \pm 1.89$ \\
\hline
\end{tabular}

All values expressed as Mean $\pm S D$, $(n=4)$; * $p<0.05$ significant, $* * p<0.01$ highly significant, $* * * p<0.001$ very highly significant. Statistical Comparision was performed by using ANOVA coupled with Dunnet's multiple comparision tests.
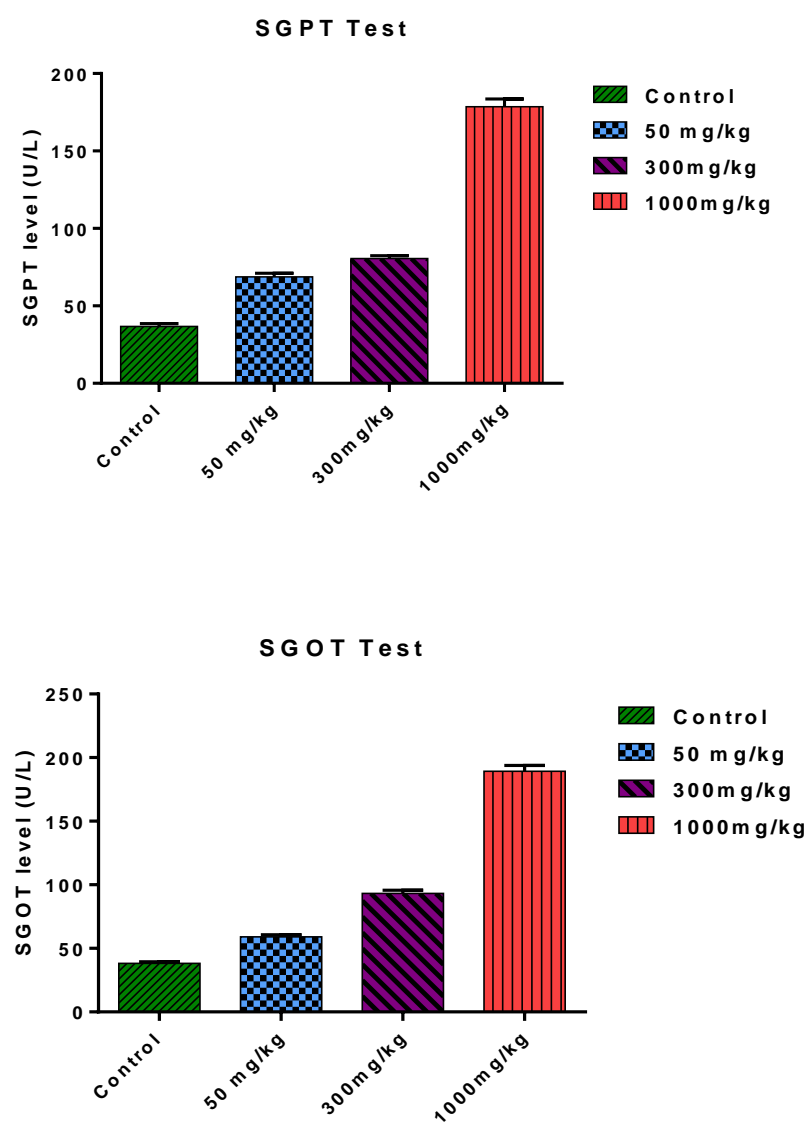

Fig 9: Liver Function Tests 
C Srinivas Reddy et al.

3. Kidney function test: serum creatinine estimation

Table 9: Serum Creatinine levels of 28 day-treated animals at various doses.

\begin{tabular}{cc}
\hline Group & Serum Creatinine $(\mathrm{mg} / \mathrm{dl})$ \\
\hline $1000 \mathrm{mg} / \mathrm{kg}$ & $3.06 \pm 0.33^{* * *}$ \\
$300 \mathrm{mg} / \mathrm{kg}$ & $2.28 \pm 0.19^{* *}$ \\
$50 \mathrm{mg} / \mathrm{kg}$ & $1.75 \pm 0.10^{*}$ \\
Control & $0.89 \pm 0.01$ \\
\hline
\end{tabular}

All values expressed as Mean $\pm \mathrm{SD},(\mathrm{n}=4) ;{ }^{*} \mathrm{p}<0.05$ significant, ${ }^{*} \mathrm{p}<<0.01$ highly significant, $* * * \mathrm{p}<0.001$ very highly significant. Statistical Comparision was performed by using ANOVA coupled with Dunnet's multiple comparision test.

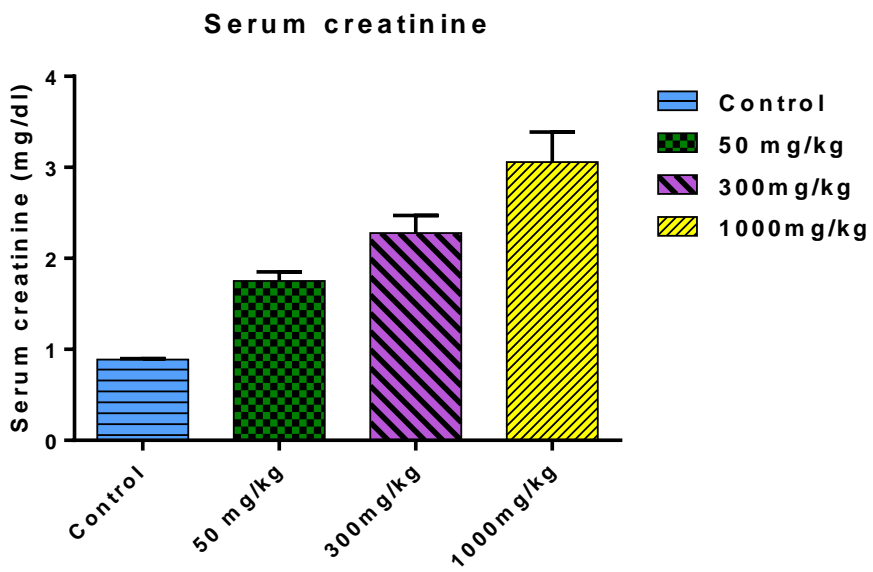

Fig 10: Kidney Function Test 


\section{E. HISTOPATHOLOGICAL STUDIES}

\section{Fig: 11 HISTOPATHOLOGY OF LIVER}
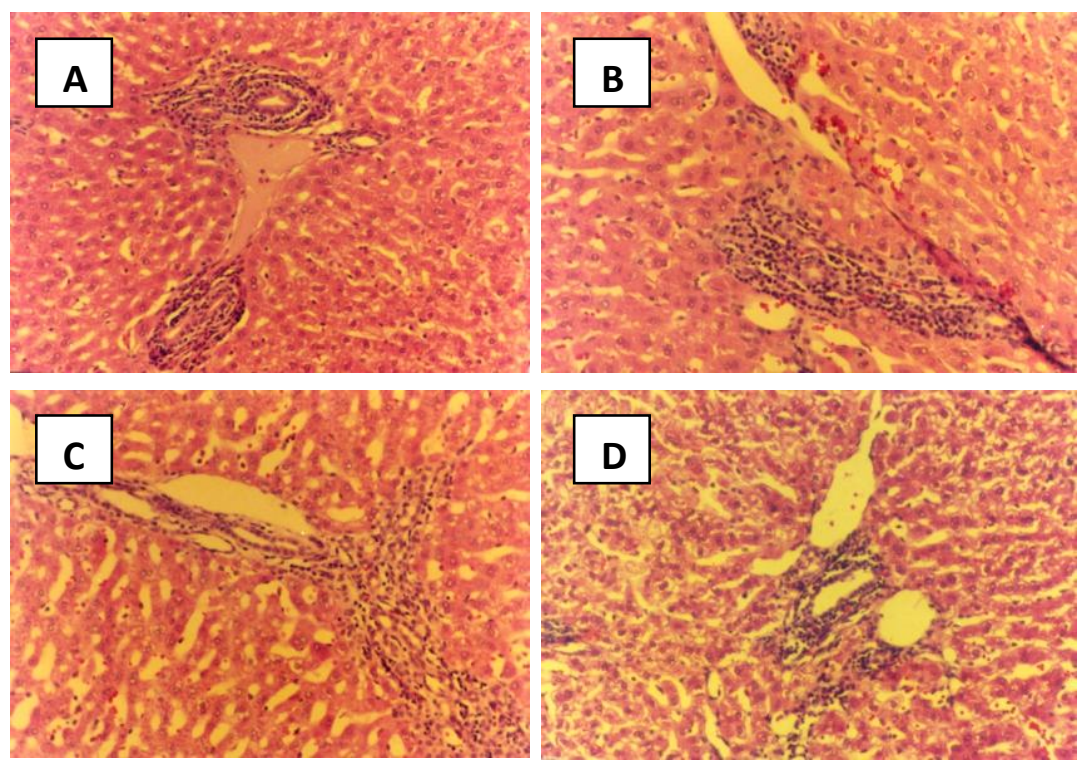

Control (A): Liver showing normal architecture

Low dose (B): Showing mild degenerative changes with few focal mono nuclear cell infiltration

Medium dose (C) showing mild-moderate degenerative changes with dilatation of hepatic venule with focal mono nuclear cell infiltration

High dose (D): showing dilated bile duct and portal tract with a proliferative changes and occasional necrotic changes

Fig. 12: HISTOPATHOLOGY OF KIDNEY
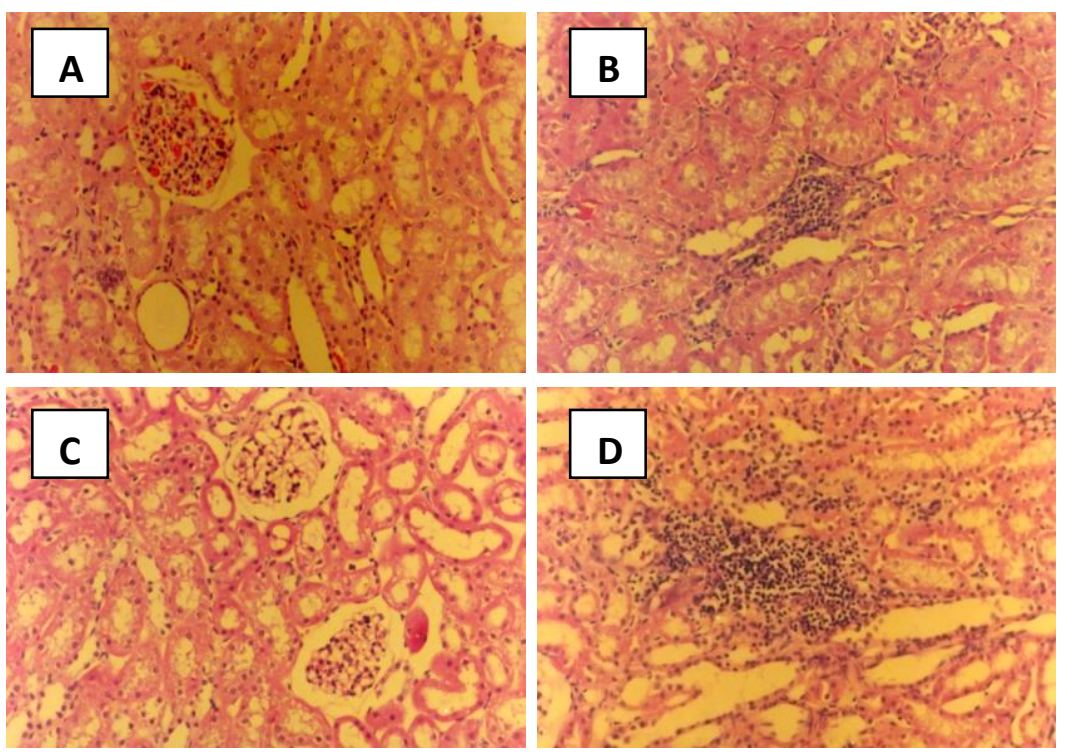

Control (A): Showing normal renal cortical parenchyma

Low dose (B): Showing normal renal cortical parenchyma with focal mild mono nuclear infiltration in Bowman's capsule

Medium dose (C): Shows mild degenerative changes with mono nuclear infiltration in the renal parenchyma

High dose (D): Show diffuse infiltration of mono nuclear cells with focal necrotic debris 
Fig. 13: HISTOPATHOLOGY OF SPLEEN
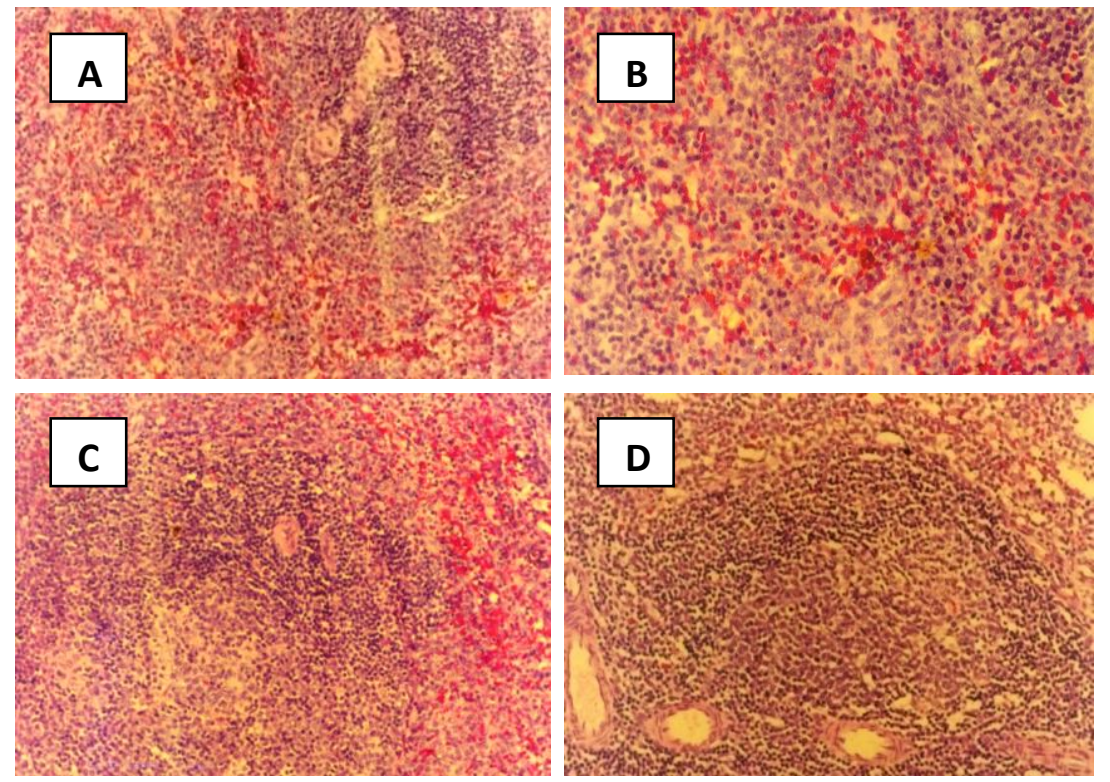

Control (A): Shows normal lymphoid masses (White pulp)

Low dose (B): Shows mild proliferative changes in the sphlenic lymphoid masses

Medium dose (C): Shows mild-moderate proliferative changes in the sphlenic lymphoid masses High dose (D): Spleen showing mild-moderate proliferative changes in the sphlenic lymphoid masses

Fig. 14: HISTOPATHOLOGY OF LUNG
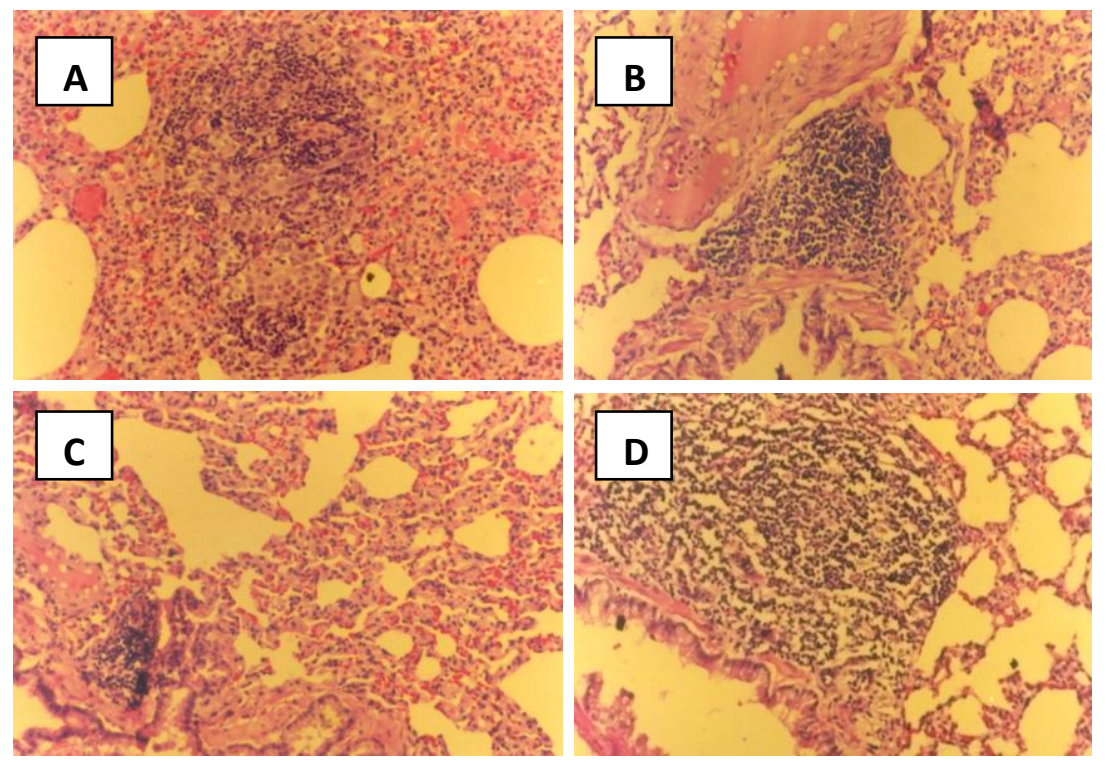

Control (A): Shows normal bronchioles and alveoli

Low dose (B): shows alveoli with mild inflammatory mono nuclear infiltration

Medium dose (C): Shows foci of mono nuclear aggregates in the lung parenchyma

High dose (D): the alveoli adjacent to bronchiole a large aggregates of mono nuclear cell infiltration and no degenerative changes 

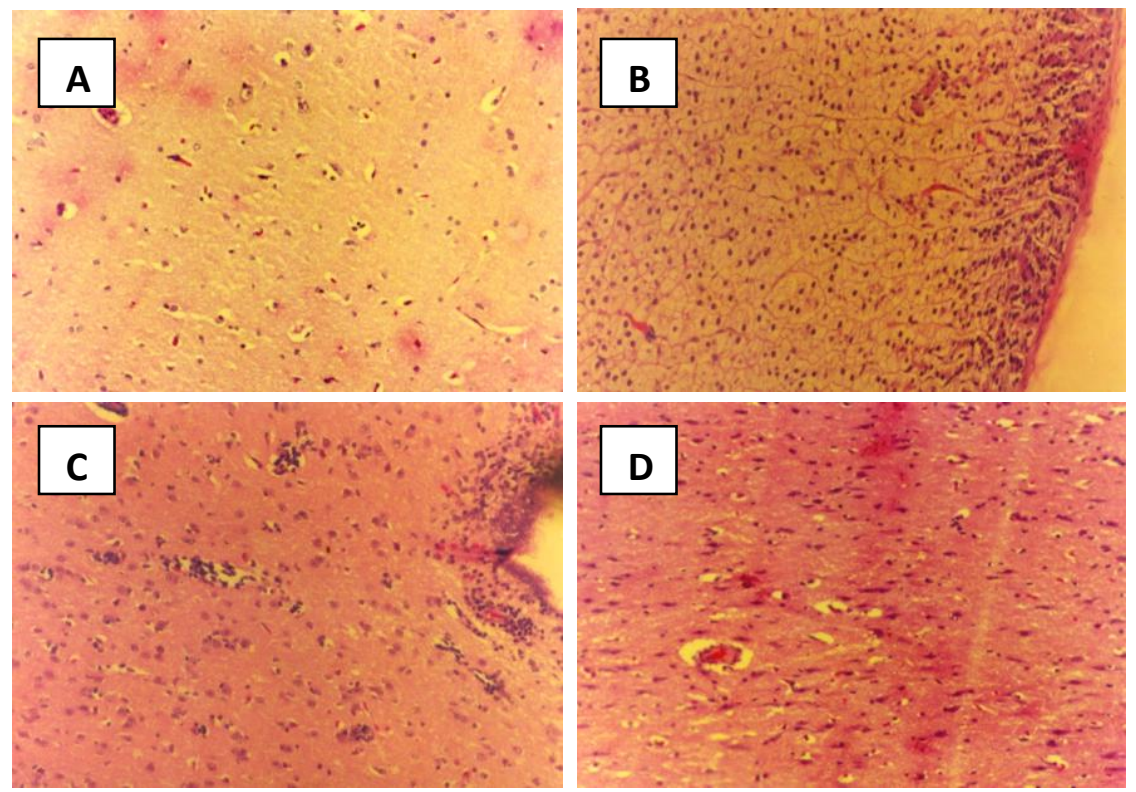

Control (A): Shows normal white matter of cerebral cortex

Low dose (B): shows normal white matter of cerebral cortex Medium dose (C): Shows normal white matter of cerebral cortex

High dose (D): Shows normal white matter of cerebral cortex

\section{DISCUSSION}

Repeated dose toxicity studies categorically suggest that with increasing doses there were significant changes taken place in the clinical/ biochemical parameters evaluated. Though the changes are not intense, but changes occurred in the complete blood picture were significant at higher doses. The elevated serum creatinine levels indicating an extensive damage to the kidney at higher doses. The glomeruli in the kidneys are those which were extensively damaged and inflammation mediated necrotic tissues were mainly seen in the medium and high dose study groups. A significant damage to the liver is indicated by the elevations in the SGPT and SGOT levels. The changes in the clinical/ biochemical parameters were strongly supported by the histopathological studies. There was also level II (medium) damage in lungs and liver in higher repetitive doses.

There was a strong positive correlation between the clinical parameters, histopathological studies and the tissue concentrations in the study groups which is pivotal for these present investigations. Mercury concentrations in the kidney are very high followed by the liver and lungs. Some accumulation of mercury is also observed in spleen and the least content observed in brain. There was 15 to 20 fold increase in the kidney mercury concentrations in high dose compared to low dose groups. Tissue to blood concentrations is also high, suggesting a strong association and high affinity binding that led to damage of glomeruli leading to elevation in creatinine levels. In liver there was 5 fold increase in mercury levels between the low dose and high dose groups and hence necrosis in the hepatocytes and subsequent elevation in SGPT and SGOT levels. 
There was dose dependent increase in the plasma concentrations. From the toxicokinetic parameters, Plasma clearance (CL) was extremely low with high half life $\left(\mathrm{T}_{1 / 2}\right)$. This strongly correlates with the high plasma protein binding/affinity of mercury. Increase in AUC and $\mathrm{V}_{\mathrm{d}}$ was also observed with increasing doses. But it is quite evident from the study that plasma concentrations and subsequent PK analysis doesn't supinely speak about the toxicity of mercury as evident from the tissue concentrations even at low doses. Saturation kinetics of the mercury at high dose needs further investigations.

\section{CONCLUSION}

Above investigations categorically conclude that low single dose or even low repeated dose of mercury could be safe and may not lead to accumulation in the tissues especially in kidney - the target organ prone to damage. Medium dose administration needs stringent monitoring of the clinical/biochemical parameters for optimization of the dose. At this dose the administration of the drug or its continuation is at the discretion of the medical practitioner. High single dose and high repeated doses have showed very deleterious effects, further continuation may lead to severe nephrotoxicity, thus shila sindur at higher doses are not advisable for administration. Therefore, the process of purification and preparation of the product-shila sindur need to be improved.

\section{REFERENCES}

1. Anand PR. Enjoy long and healthy life with Mirzam Josh, Marjoram (Majorana Hortensis Moench.) Ayurveda for all, 2008, 5; 40-42.

2. Anand PR. Resveratrol - An unique solution for all health problems, environmental science engineering, 2008, 6, 23-26.

3. Galib, Mayur B, Mayur M, Chandrashekhar J, Patgiri B J, Prajapati P K. Therapeutic Potentials of Metals in Ancient India: A review through Charaka Samhita, Journal of Ayurveda and Integrative Medicine, 2011; 2(2): 55-63.

4. Paul I D. Heavy metal poisoning from Ayurvedic traditional medicines: an emerging problem? International Journal of Environment and Health, 2008; 2(3): 463-473.

5. Robert B S, Stefanos N K, Janet P, Michael J B, David M. E, Roger B. D, Russell S P. Heavy Metal Content of Ayurvedic Herbal Medicine Products. Journal of American Medical Association, 2004; 292(23): 2868-2873.

6. Sudhakar Gurpur K, Verma R, Vijaya Bhasker K, Kumar Lalit. Heavy metals in environment, living systems and herbal preparations- An Overview. International research journal of pharmacy, 2012; 3(7): 128-130.

7. Farzana Z, Shamim J R, Soghra K H, Rizwan H K. Low dose mercury toxicity and human health. Environmental toxicology and pharmacology, 2005; 03: 1-10.

8. Srilakshmi D, Swetha S, Minchitha KU and Maheshwari KS. Shila sindura: an antimicrobial agent. International Journal of Research in Ayurveda and Pharmacy, 2012; 3(5): 671-75.

9. OECD, Test guideline 417: Toxicokinetics. OECD guidelines for the testing of chemicals (1984).

Challa Srinivas Reddy ${ }^{*}$, Umamahesh Balekari, Vemu Priyanka, Jinugu Vidya Sagar. Toxicokinetic and Tissue distribution studies of Mercury in an Ayurvedic preparation- Shila Sindur .Hygeia.J.D.Med 2014; 6(1):81-94. Available from http://www.hygeiajournal.com / Article ID- Hygeia.J.D.Med/125/14. D.O.I: 10.15254/H.J.D.Med.6.2014.125 